\title{
Monitoring Oxygenation
}

K. K. Tremper, T. W. Rutter and J. A. Wahr

The most basic goal of anaesthetists and intensivists is to assure that there is an adequate supply of oxygen to the mitochondria throughout the body to maintain aerobic metabolism and cellular function. The determination of adequate oxygenation has historically been monitored by the absence of organ dysfunction because techniques were not available to assess quantitatively the oxygenation of tissues. Over the past $\mathbf{4 0}$ years, measurement and monitoring methods have been developed to first quantitate oxygen supply and more recently to continuously asess both invasively and noninvasively the oxygenation of blood and tissue.' This article will review the current status of the invasive and noninvasive techniques for monitoring oxygenation.

\section{Oxygen tension measurement \\ Clark $\mathrm{PO}_{2}$ electrode}

In 1956, Leland Clark developed the polarographic oxygen electrode for measuring oxygen partial pressure. ${ }^{2}$ With the addition of the Severinghaus carbon dioxide electrode in 1958 , the blood gas machine was developed and critical patient care was revolutionized. ${ }^{3}$

The Clark electrode is an electrical cell composed of a platinum cathode and a silver anode. As in any resistive circuit as the voltage is increased the current will also increase. In this electrochemical cell there is a plateau voltage range over which the current does not increase with voltage but does increase with oxygen tension in the cell. An oxygen consuming electrochemical reaction takes place at the cathode, and the electric current in the circuit is directly

Kevin K. Tremper PhD MD Professor and Chair, Timothy $W$. Rutter MD Associate Professor and Associate Chair for Clinical Affairs, Joyce A. Wahr MD Assistant Professor, Department of Anesthesiology, University of Michigan, Ann Arbor, Michigan, USA. proportional to the oxygen consumed at the cathode. This cell is covered with a membrane which is freely permeable to oxygen. Clark's polarographic oxygen electrode has been used for over 30 years to measure oxygen partial pressure in gases and liquids in both medicine and industry.

\section{$\mathrm{PO}_{2}$ optode}

Just as electrochemical sensors quantify the concentration of a substance on the basis of electrochemical (voltage or current) changes, optodes quantify concentrations on the basis of photochemical reactions which produce changes in light absorption, light reflection or light emission (luminescence or fluorescence). When photons of light strike a substance, the light can either be transmitted, reflected, absorbed, or absorbed and re-emitted. ${ }^{4}$ Substances which reemit absorbed light are termed fluorescent, phosphorescent, or luminescent depending on the photochemistry of the dye involved. ${ }^{5}$ When the energy of a photon is absorbed by a luminescent dye, electrons in the dye are elevated to a higher energy state. After a small time lag, a photon of light is 


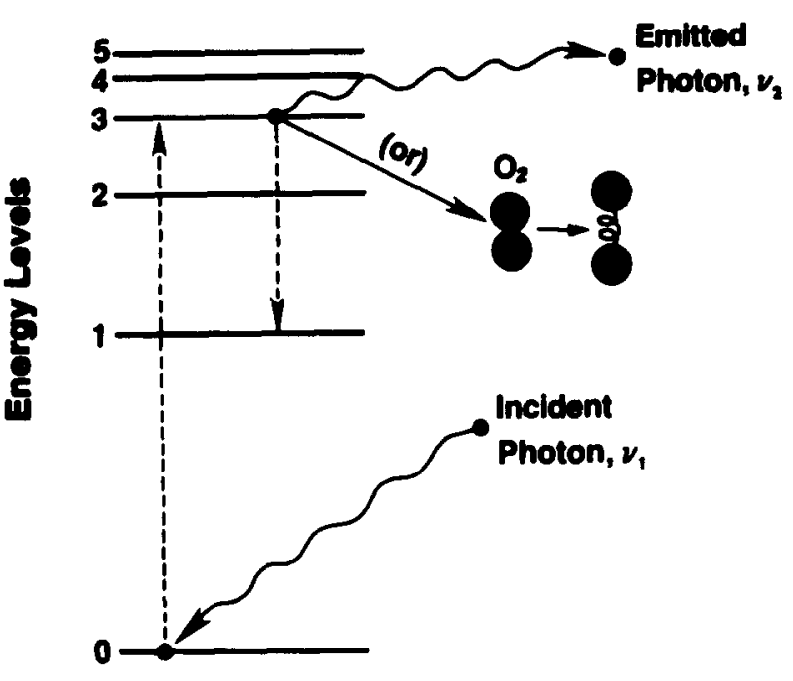

Fig. 1 - The fluorescence quenching phenomenon. An electron of the fluorescent dye is excited to a higher energy level by an incident photon $\left(v_{1}\right)$. This excited electron can return to a lower energy level either by emitting a photon $\left(v_{2}\right)$, or by interacting with an oxygen molecule and raising the latter to a higher energy level. (From: Barker SJ,

Tremper KK. Continuous fiberoptic arterial oxygen tension measurements in dogs. J Clin Monit 1987; 3: 48-52, with permission.)

re-emitted as the electron drops back to its ground state (Fig. 1). In certain dyes this re-emitted energy can be absorbed by oxygen molecules, 'quenching' the intensity of the luminescent signal. ${ }^{5.6}$ The intensity of the luminescent signal reliably decreases as the concentration of oxygen increases. The theoretical relationship between luminescent intensity and oxygen concentration is given by the Stern Vulmar equation:

$$
\mathrm{L}_{n_{\mathrm{L}}}=\mathrm{KPO}_{2}+1
$$

where $\mathrm{L}_{O}$ is the intensity of the luminescent signal in the absence of oxygen, $\mathrm{L}$ is the intensity of the signal in the presence of oxygen, and $\mathrm{K}$ is a quenching constant. Equation 1 can be rearranged to the following:

$$
\mathrm{PO}_{2}=\left[\left(\mathrm{Lo}_{\mathrm{L}}\right)-1\right] / \mathrm{K}
$$

The degree of change in the intensity of luminescent light is much greater at low concentrations of oxygen than at higher ones, indicating that the luminescent quenching techniques should be more accurate at low oxygen tensions. The luminescence quenching method for measurement of oxygen tension has been known for years but its application with optical fibres to produce oxygen sensors has come about only in the past decade. ${ }^{7}$ An oxygen probe can be constructed by placing an oxygen sensitive dye (with a known quenching constant, $K$ ) at the tip of a fibreoptic strand. A xenon lamp emits light of a known intensity down the fibreoptic strand, and the dye re-emits an intensity of light back up the strand inversely proportional to the oxygen tension surrounding the dye.
$\mathrm{PO}_{2}$ optodes were initially based upon quenching of luminescence intensity as described above. A second means of determining partial pressure of oxygen with optodes is based on the known time lag from excitation to re-emission of the photon, known as the 'luminescence lifetime'. ${ }^{8}$ This 'lifetime' is reliably altered in the presence of oxygen, and $\mathrm{PO}_{2}$ therefore can be determined by pulsing incident light and measuring the shift in luminescence lifetime. The equation describing the relationship between $\mathrm{PO}_{2}$ and the lifetime shift is:

$$
\mathrm{PO}_{2}=\left[\left(\mathrm{t}_{\mathrm{o}} / \mathrm{t}\right)-1\right] / \mathrm{k}
$$

where $t_{O}$ is the luminescence lifetime in the absence of oxygen, $t$ is the luminescence lifetime in the presence of oxygen, and $\mathrm{k}$ is a luminescence time constant. The theoretical advantage of using a 'time shift' method to measure $\mathrm{PO}_{2}$ versus intensity quenching is that intensities of light can be distorted not only by oxygen quenching, but also by bending of the optical fibre, dye photo bleaching and misalignment of optical connectors; time shift measurements are not affected by these practical considerations. When luminescence intensity is used, a second dye is required whose luminescence is unaffected by the presence of oxygen, thus providing a reference intensity to account for the losses of light intensity due to fibreoptic transmission characteristics.

Because of the inherent characteristics of fibreoptics and photochemical dyes, fluorescent optodes offer a number of advantages over other possible sensor designs. They are uniquely suited for miniaturization because of the availability of very small diameter yet reliable optical fibres (as small as $50-100 \mu \mathrm{m}$ ). The dyes used to measure gas tensions are stable over time, are biocompatible (non-toxic to the patient, and not affected by the patient environment), have a fast response time to changes in concentration of the measured substance $(60-90 \mathrm{~s})$ and are not consumed by the measurement process. The probe assembly is relatively simple and fairly immune to interference from the environment. Despite the inherent simplicity of optodes, the geometry of the optical fibres and the characteristics of the photochemical dye substances have posed substantial technical problems which have been addressed in a variety of ways by manufacturers of these devices.

\section{Haemoglobin saturation measurement}

Adult blood usually contains 4 species of haemoglobin: oxyhaemoglobin $\left(\mathrm{O}_{2} \mathrm{Hb}\right)$, reduced haemoglobin (Hb), methaemoglobin (MetHb), and carboxyhaemoglobin ( $\mathrm{COHb})$. $\mathrm{COHb}$ and $\mathrm{MetHb}$ are found in low concentrations except in pathologic states. Because these dyshaemoglobins do not transport oxygen, they do not contribute to the oxygen 

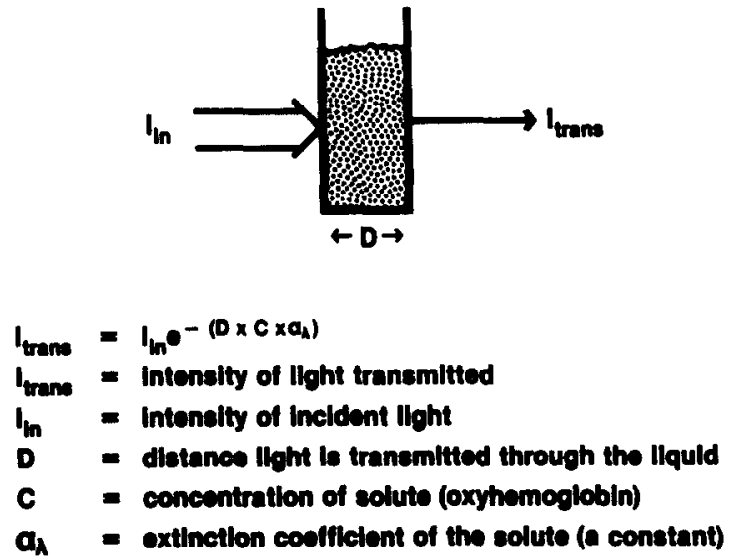

Fig. 2 - The concentration of a solute dissolved in a solvent can be calculated from the logarithmic relationship between the incident and transmitted light intensity and the solute concentration. (From: Tremper KK, Barker SJ. Pulse oximetry and oxygen transport. In: Payne JP, Severinghaus JW, eds. Pulse Oximetry. Berlin: Springer-Verlag, 1986: 19-27, with permission.)

content. The term 'functional' haemoglobin saturation is defined as:

$$
\text { Functional } \mathrm{SaO}_{2}=\frac{\mathrm{HbO}_{2}}{\mathrm{HbO}_{2}+\mathrm{Hb}} \times 100 \% \quad \text { eq. } 4
$$

This definition of haemoglobin saturation does not include $\mathrm{MetHb}$ or $\mathrm{COHb}$ because they do not contribute to oxygen transport. Fractional haemoglobin saturation, which is also called oxyhaemoglobin fraction, is defined as:

$$
\text { Fractional } \mathrm{SaO}_{2}
$$

$$
=\frac{\mathrm{HbO}_{2}}{\mathrm{HbO}_{2}+\mathrm{Hb}+\mathrm{COHb}+\mathrm{MetHb}} \times 100 \% \text { eq. } 5
$$

This definition of haemoglobin saturation, that is the ratio of oxyhaemoglobin to total haemoglobin in the saturation used in the calculation of oxygen content and delivery. It is important to remember these definitions when evaluating the clinical utility and limitations of haemoglobin saturation monitors.

\section{Beer's law}

Spectrophotometry was first used to determine the haemoglobin concentration of blood in the 1930s. ${ }^{4}$ This method is based on the Lambert-Beer law, which relates the concentration of a solute to the intensity of light transmitted through the solution (Fig. 2):

$$
\mathrm{I}_{\text {trans }}=\mathrm{I}_{\mathrm{in}} \mathrm{c}^{-\mathrm{DC}} \alpha
$$

where

$I_{\text {trans }}=$ intensity of transmitted light

$\mathrm{I}_{\text {in }}=$ intensity of incident light

$\mathrm{D}=$ distance light is transmitted through the liquid

$\mathrm{C}=$ concentration of the solute (haemoglobin) $\alpha=$ extinction coefficient of the solute

(a constant for a given solute

at a specific light wavelength)*

Thus if a known solute is dissolved in a clear solvent in a cuvette of known dimensions, the solute concentration can be calculated if the incident and transmitted light intensity are measured (Fig. 2). The extinction coefficient is independent of the concentration, but is a function of the light wavelength used.

( ${ }^{*}$ This law further states that the absorbance from multiple solutes in solution is the sum of the absorbances of the various solutes times their respective concentrations, e.g.: $\mathrm{C}_{11}+\mathrm{C}_{22}+\mathrm{C}_{33}$, etc.)

Laboratory oximeters use this principle to determine haemoglobin concentration by measuring the intensity of light transmitted through a haemoglobin dispersion produced from lysed red blood cells. For each wavelength of light used an independent Lambert-Beer equation can be written. If the number of equations is equal to the number of solutes (i.e. haemoglobin species) then we can solve for the concentration of each use. Therefore, to determine the concentrations of four species of haemoglobin we require at least 4 wavelengths of light. For the Lambert-Beer to be valid, both the solvent and cuvette must be transparent at the light wavelengths used, the light path length must be known exactly, and no other absorbers can be present in the solution. It is difficult to fulfil all of these requirements in clinical devices. Consequently, although these devices are theoretically based on the Lambert-Beer law, they also require empirical corrections to improve their accuracy.

\section{Continuous oxygen tension monitoring}

\section{Invasive $\mathrm{PO}_{2}$ monitoring: Clark electrode}

The primary problem in continuous invasive $\mathrm{PaO}_{2}$ monitoring is miniaturization of the Clark electrode to permit insertion through an arterial cannula. There are two approaches to this problem. One is to insert only the platinum cathode in the arterial cannula and place the reference anode on the skin surface. The platinum cathode is surrounded by a thin layer of electrolyte and covered with an oxygen permeable membrane. ${ }^{10}$ The second approach involves miniaturization of the entire anode-cathode electrode for intraarterial insertion. ${ }^{11,12}$

There have been several studies of intraarterial $\mathrm{PO}_{2}$ monitoring using Clark electrodes, and the results are somewhat conflicting with respect to accuracy. It is often difficult to compare such studies because the data are usually analysed by linear regression and correlation coefficients. Altman et al have shown that this may be inappropriate and often misleading in methods-comparison studies. ${ }^{13}$ The correlation coefficient is extremely sensitive to the $x$ and $\mathrm{y}$ range over which the data are collected. 
Furthermore, a high correlation coefficient ( $\mathrm{r}$ close to 1.0) implies a high degree of association between the methods (i.e. when one goes up the other will go up) but it does not imply that one method can replace the other. As an alternative, Altman recommends using the mean and standard deviation of the difference between the two methods of measurement as an assessment of agreement. The mean difference is called the 'bias' and the standard deviation is referred to as the 'precision'. The bias indicates the systematic error, i.e. the consistent over or underestimate of one method relative to the other while the precision represents the scatter or random error.

Umbilical artery Clark electrodes in neonates have been associated with a number of complications, including thrombus formation, embolism, vascular perforation, infarction, lower extremity ischaemia, and infection. The size of the electrode also causes problems with blood pressure monitoring and arterial blood sampling. Other reported problems include calibration drift and systematic underestimation of $\mathrm{PaO}_{2}$. The causes of these errors are not understood, but they may involve decreased blood flow around the electrode tip or clot formation on the electrode surface.

Because of the difficulties in miniaturizing both the Clark electrode and the glass components required for electrochemical $\mathrm{CO}_{2}$ and $\mathrm{pH}$ measurement, it is unlikely that an electrochemical blood gas monitoring system will ever be developed for intraarterial use. The optode technology is therefore being developed for this application.

\section{Optode clinical studies}

In spite of the enthusiasm over intravascular blood gas monitoring with optodes, there are few clinical studies to date to assess accuracy, a few case reports which may demonstrate utility and no data which address cost benefit issues. The first clinical study on a triple function optode was presented by Shapiro et al in $1989 .{ }^{14}$ This study was conducted in dogs and patients. The probe was $0.62 \mathrm{~mm}$ diameter optode produced by CDI 3-M Corporation and was inserted through a 20 gauge arterial catheter. In this first study all optodes were in vivo calibrated to a bench blood gas analyser. 79 blood samples were collected from 12 critically ill patients over a period of time ranging from $3-26 \mathrm{~h}$. This study was terminated due to a system fault in 5 of the 12 patients. The bias and precision data are $-1.22 \pm 9.34 \mathrm{mmHg}$. The bias data look good, but all of these devices were in vivo calibrated with a bench blood gas analyser first, and 13 probes were eliminated from the study because they did not calibrate. Of the 12 probes that did calibrate, nearly $40 \%(5 / 12)$ failed during the study and were dropped from the analysis. The authors concluded that further study was warranted. ${ }^{14}$

A problem which appeared to plague early clinical studies was the occurrence of acute drops in the oxygen optode measurement unrelated to arterial $\mathrm{PO}_{2}$ changes. Mahutte et al ${ }^{15}$ studied oxygen optode probes in 18 volunteers to elucidate the cause of these rapid and unpredictable drops in $\mathrm{PO}_{2}$ measurement. Thcy manipulated wrist position and sensor position and varied the inspired oxygen and carbon dioxide concentrations. They first found that clot formation at the tip of the sensor caused a 'down-up-down' pattern with decreased $\mathrm{PO}_{2}$, increased $\mathrm{PCO}_{2}$, and decreased $\mathrm{pH}$. They also demonstrated that acute drops in oxygen measurement could be produced when the probe was adjacent to the arterial vessel wall. If the probe were then slightly retracted the $\mathrm{PO}_{2}$ value would return to normal. They concluded that the distance the probe protruded beyond the cannula was critical; over-extension caused a wall effect (unpredictable drops in oxygen values), while retraction too far within the cannula produced values which were contaminated by a flush solution. To investigate this 'wall effect', they constructed a special probe with three oxygen sensors $120^{\circ}$ apart on the sides of the sensor tip. They noted that if the probe was rotated, the $\mathrm{PO}_{2}$ 'down' pattern could be produced as each sensor faced the vessel wall.

A third clinical study was conducted on patients intraoperatively using the same CDI 3-M optode by Barker and Hyatt. ${ }^{16}$ In this study the optode was inserted through an 18 gauge radial artery catheter. 87 arterial blood samples were compared to optode values; no acute drops in $\mathrm{PO}_{2}$ were noted. The following bias and precision were found: $-9 \pm 23.3$ $\mathrm{mmHg}$. Note the bias value was negative in this study. Although these data appear to be approaching clinically acceptable accuracy the necessity of using a 18 gauge catheter reduced the clinical desirability of this CDI 3-M probe.

The largest human study to date on an optode blood gas system is the multicentre trial of an ex-vivo CDI 2000 Blood Gas Monitoring System. ${ }^{17}$ This system has the sensing chamber located outside of the body. When analysis is desired, an arterial sample is withdrawn into the sensing chamber, and results are displayed after a period of approximately $2 \mathrm{~min}$. This addresses the issues of thrombogenicity and arterial wall interference, but is, again, a measurement rather than a monitoring technique. Nonetheless, response time is fast enough to permit very frequent (many times per hour) sampling while preserving test reliability and protecting both patients and personnel from risk of blood borne infections. The results from this study included 1341 samples drawn from 117 patients over a period of up to $80 \mathrm{~h}$. The bias and precision values are $-2.2 \pm 8.7 \mathrm{mmHg}$.

Zimmermann and Gellinger recently presented a study on a triple optode probe produced by Optex Corporation. ${ }^{18} 104$ blood samples from 5 critically ill patients monitored from up to $68 \mathrm{~h}$ were compared to two different bench top blood gas analysers. The bias and precision values were $-5.89 \pm 13.19 \mathrm{mmHg}$. The arterial blood samples were analysed on two different 
manufacturers of blood gas machines to assess the bias and precision of one blood gas machine versus another. The bias and precision values comparing the two blood gas machines were nearly identical to that comparing the blood gas machine to the optode sensor. ${ }^{18,19}$

In both the $\mathrm{CDI}^{17}$ and Optex ${ }^{18}$ studies probes were calibrated in vivo to laboratory determined arterial blood gases. No information is provided from either study as to the degree of correction required at each calibration (i.e. the drift). Thrombus formation during use of an indwelling probe also remains a concern which has not been adequately answered to date. In the 5 patients studies with the Optex probe, the authors state that no problems were noted with arterial wave forms, and no evidence of thrombus was seen. However, the number of patients remains small, and none of the studies in the literature specifically address thrombus formation as a study question - for example, no Doppler studies have been done following removal of the arterial cannula or probe. Sensors used in the CDI study were examined microscopically for thrombus, but no results are presented other than to state that no patients were removed from the study due to clot formation or sensor failure.

Finally, there are two case reports in the literature, one reporting the use of a CDI probe during single lung ventilation, ${ }^{20}$ the other relating detection of venous air embolism during monitoring with a CDI probe. ${ }^{21}$ These reports may indicate the applicability of these monitoring devices to specific cases.

\section{Noninvasvie $\mathrm{PO}_{2}$ monitoring: transcutaneous $\mathrm{PO}_{2}$}

Transcutaneous $\mathrm{PO}_{2}$ is the oxygen tension of heated skin. To obtain a measurable $\mathrm{PO}_{2}$ at the skin surface with a fast response time, the skin temperature must be heated to more than $43^{\circ} \mathrm{C}$. This heating causes several changes in the various layers of the skin. The stratum corneum, composed of lipid in a protein matrix, is normally a very efficient barrier to gas transport. When heated above $41^{\circ} \mathrm{C}$ the structural characteristics of this layer change, allowing oxygen to diffuse through it readily. ${ }^{22}$ In the epidermis, heating causes vasodilatation of the dermal capillaries which is said to 'arterialize' this capillary blood. The perfusion of this hyperaemic epidermal capillary bed is also dependent upon adequate blood flow to the dermal vasculature. Consequently, if the cardiac output decreases we would anticipate a decrease in skin blood flow and hence in oxygen delivery to the transcutaneous sensor. During the shock state, $\mathrm{PtcO}_{2}$ decreased with decreasing cardiac output. This effect of cardiac output on the $\mathrm{PtcO}_{2}-\mathrm{PaO}_{2}$ relationship can be quantitated in terms of a transcutaneous oxygen index:

$\mathrm{PtcO}_{2}$ index $=\mathrm{PtcO}_{2} / \mathrm{PaO}_{2}$
$\mathrm{PtcO}_{2}$ index has been used as an indicator of peripheral oxygen delivery, analogous to the alveolar-arterial $\mathrm{PO}_{2}$ gradient for the assessment of pulmonary function. ${ }^{22}$ Under stable haemodynamic conditions the normal $\mathrm{PtcO}_{2}$ index for adult patients was 0.79 , while this index fell to 0.49 when the cardiac index dropped to less than $2.2 \mathrm{~V} / \mathrm{min} / \mathrm{m}^{2}$. In the early work on newborn infants, transcutaneous $\mathrm{PO}_{2}$ was found to be similar to $\mathrm{PaO}_{2}$; i.e. the $\mathrm{PtcO}_{2}$ index was approximately 1.0. A review of the published $\mathrm{PtcO}_{2}$ values on haemodynamically stable patients in various age groups reveals that $\mathrm{PtcO}_{2}$ index decreases progressively with age from premature infants (1.0) to elderly (0.6). Glenski et al also found that $\mathrm{PtcO}_{2}$ index is relatively independent of probe location as long as the probe is on the central body rather than the extremities. ${ }^{23} \mathrm{PtcO}_{2}$ index may be slightly higher in female adult patients. $\mathrm{PtcO}_{2}$ is therefore a continuous, noninvasive measure of peripheral tissue oxygen tension. It will follow changes in $\mathrm{PaO}_{2}$ under conditions of haemodynamic stability, and it will decrease relative to arterial $\mathrm{PaO}_{2}$ as cardiac output falls below the normal range.

The limitations of $\mathrm{PtcO}_{2}$ monitoring include calibration and electrode maintenance, a 10 to $15 \mathrm{~min}$ warm-up time, the possibility of causing skin burns, and the potential interference by halothane.

\section{Noninvasive $\mathrm{PO}_{2}$ monitoring: conjunctival $\mathrm{PO}_{2}$}

When the eyes are closed the cornea receives its oxygen supply from the palpebral conjunctiva. Therefore this inner layer of the eyelid is well vascularized and has few cell layers between the capillaries and the mucous surface. The blood supply to the palpebral conjunctiva is derived from a branch of the ophthalmic artery which in turn is a branch of the ipsilateral internal carotid artery. To measure $\mathrm{PO}_{2}$ on this well-perfused surface, miniaturized Clark electrodes have been made to fit inside a polymethylmethacrylate ocular conformer ring. This conformer directly applies the Clark electrode to the inner surface of the palpebral conjunctiva. The electrode is not heated and therefore measures the surface oxygen directly from the tissue. This device is a true tissue oxygen monitor; unlike $\mathrm{PtcO}_{2}$ its values are not perturbed by heating. There are several advantages to this technique. First, since the probe is not heated it requires only $60 \mathrm{~s}$ to equilibrate with the local tissue $\mathrm{PO}_{2}$. Second, since the blood supply is from the carotid artery this monitor will help detect changes in carotid blood flow. ${ }^{24}$ Since conjunctival $\mathrm{PO}_{2}\left(\mathrm{PcjO}_{2}\right)$ is another measure of tissue oxygenation, its values will be affected by both $\mathrm{PaO}_{2}$ and cardiac output in the same manner that was described above for transcutaneous $\mathrm{PO}_{2}$. Therefore its values are also divided by arterial $\mathrm{PO}_{2}$ to yield an index analogous to the transcutaneous $\mathrm{O}_{2}$ index. The $\mathrm{PcjO}_{2}$ index is then a measure of the relative perfusion of conjunctival tissue. The normal $\mathrm{PcjO}_{2}$ index is reported to be 0.6 
to 0.7 , with lower values in elderly patients. The index progressively decreases with decreasing blood volume or cardiac output, again in a similar manner to $\mathrm{PtcO}_{2}$. Clinical studies have demonstrated a $\mathrm{PcjO}_{2}$ index of less than 0.5 is associated with a blood volume deficit of at least $15 \%$. Practical limitations of conjunctival oxygen monitoring are also the same as those of transcutaneous monitoring: electrode maintenance and calibration. Unlike $\mathrm{PtcO}_{2}$, the $\mathrm{PcjO}_{2}$ sensor does not produce burns or require a $10 \mathrm{~min}$ warm-up time, but because the monitor is placed on the eye there is a potential for injury to this sensitive tissue. Clinical studies to date have not reported serious problems with eye damage.

\section{Continuous haemoglobin saturation monitoring}

\section{Invasive haemoglobin saturation monitoring}

Mixed venous oxygen tension $\left(\mathrm{PvO}_{2}\right)$ and haemoglobin saturation $\left(\mathrm{SvO}_{2}\right)$ reflect global tissue oxygenation and the ability of the cardiopulmonary system to transport sufficient oxygen to meet body oxygen needs. Based on this physiologic argument, continous mixed venous oxygen monitoring should have great clinical utility. In 1973, a fibreoptic system was reported to accurately monitor mixed venous haemoglobin saturation in man. ${ }^{25}$ This device used optical fibres incorporated into a pulmonary artery catheter to estimate the haemoglobin saturation from a reflected light signal. Light at red and infrared wavelengths was transmitted down one set of fibreoptic channels while the reflected signal from intact circulating red cells was transmitted back via other fibres to an external photodetector. Although this first system appeared to work, it was not commercially produced due to the technical problems of inserting a pulmonary artery catheter which was made relatively stiff by the fibreoptic bundles. In the late 1970s Oximetrix, Inc. (Division of Abbott Laboratories) developed two fibreoptic reflectance systems for measuring haemoglobin saturation. The first system, introduced in 1977 , employed a $7 \mathrm{~F}$ double-lumen umbilical artery catheter to be used in monitoring critically ill newborn infants. ${ }^{26}$ The second system, introduced in 1981, used a $7.5 \mathrm{~F}$ pulmonary artery catheter with thermodilution capability for cardiac output measurement in addition to continuous mixcd venous saturation monitoring. ${ }^{27}$ These new systems used three wavelengths of light to calculate saturation. As discussed earlier a minimum of four wavelengths are required to calculate haemoglobin saturation from Beer's law in the presence of MetHb and COHb. The Oximetrix mixed venous saturation monitor can accurately measure functional haemoglobin saturation in the absence of significant dyshaemoglobin concentrations. ${ }^{27}$ However, MetHb and $\mathrm{COHb}$ pro- duce significant errors in the saturation measurement. ${ }^{28,29}$ (This will be discussed further in the section on pulse oximetry.) American Edwards Corporation has also produced a mixed venous saturation pulmonary artery catheter which uses two wavelengths of light. This device requires manual entry of the total haemoglobin to improve accuracy, whereas the Oximetrix system can accurately measure mixed venous saturation over a wide range of haematocrits. ${ }^{30}$ Although in theory, continuous monitoiring of mixed venous saturation would appear to be a useful variable to follow in the critically ill, its actual clinical utility is controversial. ${ }^{31,32}$ In this age of cost benefit as well as risk benefit each device will have to justify its value in patient management.

\section{Noninvasive haemoglobin saturation monitoring: pulse oximetry}

In only a few years after its introduction to the operating room, the pulse oximeter has become an anaesthetic standard of care. ${ }^{33}$ This technique has been rapidly accepted by the anaesthesia community because it continuously and noninvasively monitors the arterial oxygenation with little user effort. This section will discuss the theoretical principles upon which pulse oximetry is based, its technical and physiologic limitations, and its clinical applications.

Technical development of pulse oximetry. The term oximeter was coined by Glen Millikan who in the 1940s developed a lightweight device for noninvasively monitoring haemoglobin saturation. ${ }^{34}$ This device estimated haemoglobin saturation by transilluminating the ear with light of two wavelengths, one in the red and one in the infrared range. The transmitted light was measured with a photodetector. This Beer's law device effectively used the earlobe as a cuvette containing haemoglobin (Fig. 2). The two primary technical problems involved estimating arterial haemoglobin saturation in living tissue rather than in vitro. First, there are many light absorbers in the tissues other than haemoglobin. Second, the tissues contain not only arterial blood, but also venous and capillary blood. Millikan approached these problems by first measuring the absorbance of the ear while it was compressed to eliminate the blood. After this bloodless baseline measurement, the ear was heated to 'arterilize' the blood. The difference between this absorbent signal and the baseline value was thus related to the arterial blood. This device was demonstrated to accurately detect intraoperative desaturations in the early 1950s, but because of technical difficulties with its use it was never adopted as a routine clinical monitor.

In the mid 1970s, a Japanese engineer named Takuo Aoyagi made an ingenious discovery. $\mathrm{He}$ noted that the pulsatile components of the absorbances of red and infrared light transmitted through 




$R=\frac{A C_{660} / D C_{600}}{A C_{940} / D C_{940}}$

Fig. 3 - A typical oximeter calibration curve. Note that arterial oxygen saturation $\left(\mathrm{SaO}_{2}\right)$ is estimated from the ratio (R) of the pulse-added red absorbance at $660 \mathrm{~nm}\left(\mathrm{AC}_{660}\right)$ $\mathrm{DC}_{660}$ ) to the pulse-added infrared absorbance at $940 \mathrm{~nm}$ $\left(\mathrm{AC}_{940} / \mathrm{DC}_{940}\right)$. The ratios of red to infrared absorbances vary from approximately 0.4 to $100 \%$ saturation to 3.4 at $0 \%$ saturation. Note also that the ratio of red to infrared absorbance is 1.0 at a saturation of approximately $85 \%$. Although approximate determinations of this curve can be made on a theoretical basis, accurate predictions of saturation by the pulse oximeter $\left(\mathrm{SpO}_{2}\right)$ require experimental data. (From: Tremper KK, Barker SJ. Pulse oximetry. Anesthesiology 1989; 70: 98-108, with permission.)

tissue were related to arterial haemoglobin saturation. ${ }^{34}$ This eliminated the need to heat the tissue to obtain an arterial estimate. He used two wavelengths of light, one in the red (660 nanometers) and one in the infrared ( 940 nanometers). Since this device relies on the detection of a pulsatile signal it is referred to as a 'pulse oximeter'. All pulse oximeters assume that the only pulsatile absorbance between the light source and the photodetector is that of arterial blood. The pulse oximeter first determines the AC component of the absorbance at each wavelength and then divides this by the corresponding DC component to obtain a 'pulse-added' absorbance that is independent of the incident light intensity. It then calculates the ratio of the pulse-added absorbances at the two wavelengths, which is then empirically related to $\mathrm{SaO}_{2}$ :

$$
\mathrm{R}-\frac{\mathrm{AC} \text { IR/DC IR }}{\mathrm{AC} \mathrm{Red/DC} \mathrm{Red}}
$$

Figure 3 is an example of a pulse oximeter calibration curve. These curves are developed by measuring the pulse oximeter absorbance ratio $\mathrm{R}$ in human volunteers and simultaneously sampling arterial blood for in vitro saturation measurements. The ratio of absorbances $\mathrm{R}$ varies from 0.4 to 3.4 over the saturation range of 0 to $100 \%$. Note that an absorbance ratio of 1.0 corresponds to a pulse oximeter saturation $\left(\mathrm{SpO}_{2}\right)$ of approximately $85 \%$. We shall make use of this fact in the discussion below.
Although the theory of the pulse oximeter is relatively straightforward, the application of this theory to a clinically useful device engenders technical problems and physiologic limitations. One of the limitations is a consequence of Beer's law and the definitions of functional and fractional haemoglobin saturation. Being a two wavelength device, the pulse oximeter assumes that there are only two absorbing haemoglobin species in the blood stream: oxyhaemoglobin and reduced haemoglobin. If methaemoglobin or carboxyhaemoglobin are present, they will contribute to the pulse-added absorbance signal and be interpreted as either $\mathrm{O}_{2} \mathrm{Hb}$ or $\mathrm{Hb}$ or some combination of the two. We can gain some insight into the effects of dyshaemoglobins on the pulse oximeter by examining the absorbance spectra (Fig. 4). Note that $\mathrm{COHb}$ absorbs very little light at $940 \mathrm{~nm}$ (infrared), while at $660 \mathrm{~nm}$ (red light) it absorbs as much as does $\mathrm{O}_{2} \mathrm{Hb}$. This is clinically illustrated by the fact that patients with carboxyhaemoglobin toxicity appear red. The effects of $\mathrm{COHb}$ on pulse oximeter $\mathrm{SpO}_{2}$ values have been evaluated experimentally and found that $\mathrm{SpO}_{2}$ was roughly given by $\mathrm{O}_{2} \mathrm{Hb}+0.9 \times$ COHb. ${ }^{34}$

In the case of methaemoglobin, the absorbance is high at both the red and infrared wavelengths. Consequently MetHb will produce a large pulseadded absorbance at both wavelengths used by the pulse oximeter. Adding a large absorbance to both the numerator and denominator of the absorbance ratio $R$ would tend to force this ratio toward unity. On the pulse oximeter calibration curve (Fig. 3) a ratio of 1 corresponds to a saturation of $85 \%$ as noted above. The effects of methaemoglobinaemia have also been evaluated experimentally, confirming the hypothesis that high MetHb levels will force $\mathrm{SpO}_{2}$ toward $85 \%$ regardless of the $\mathrm{PaO}_{2}$ and $\mathrm{SaO}_{2}$ values. ${ }^{34}$

Any substance which absorbs light at the red and infrared pulse oximeter wavelengths and is present in the pulsatile arterial blood may cause errors. A clinical example of this effect is given by intravenously injected dyes. Scheller et al evaluated the effects of bolus injections of methylene blue, indigo carmine, and indocyanine green on pulse oximeter $\mathrm{SpO}_{2}$ values in volunteers. They found that methylene blue caused a fall in $\mathrm{SpO}_{2}$ to approximately $65 \%$ for $1-2 \mathrm{~min}$, while indigo carmine produced a very slight decrease. Indocyanine green had an intermediate effect.

With the adoption of pulse oximetry in the neonatal intensive care unit, we must consider the accuracy of the pulse oximeter in the presence of fetal haemoglobin (HbF). Fetal haemoglobin has a greater affinity for oxygen (a lower $\mathrm{P}_{50}$ ) than adult haemoglobin ( $\mathrm{HbA})$, thus it will become fully saturated at a lower $\mathrm{PaO}_{2}$. The absorption coefficients for $\mathrm{HbF}$ are no different than those of $\mathrm{HbA}$, however, so the presence of fetal haemoglobin should not cause an error in pulse oximeter saturation estimates. Since 


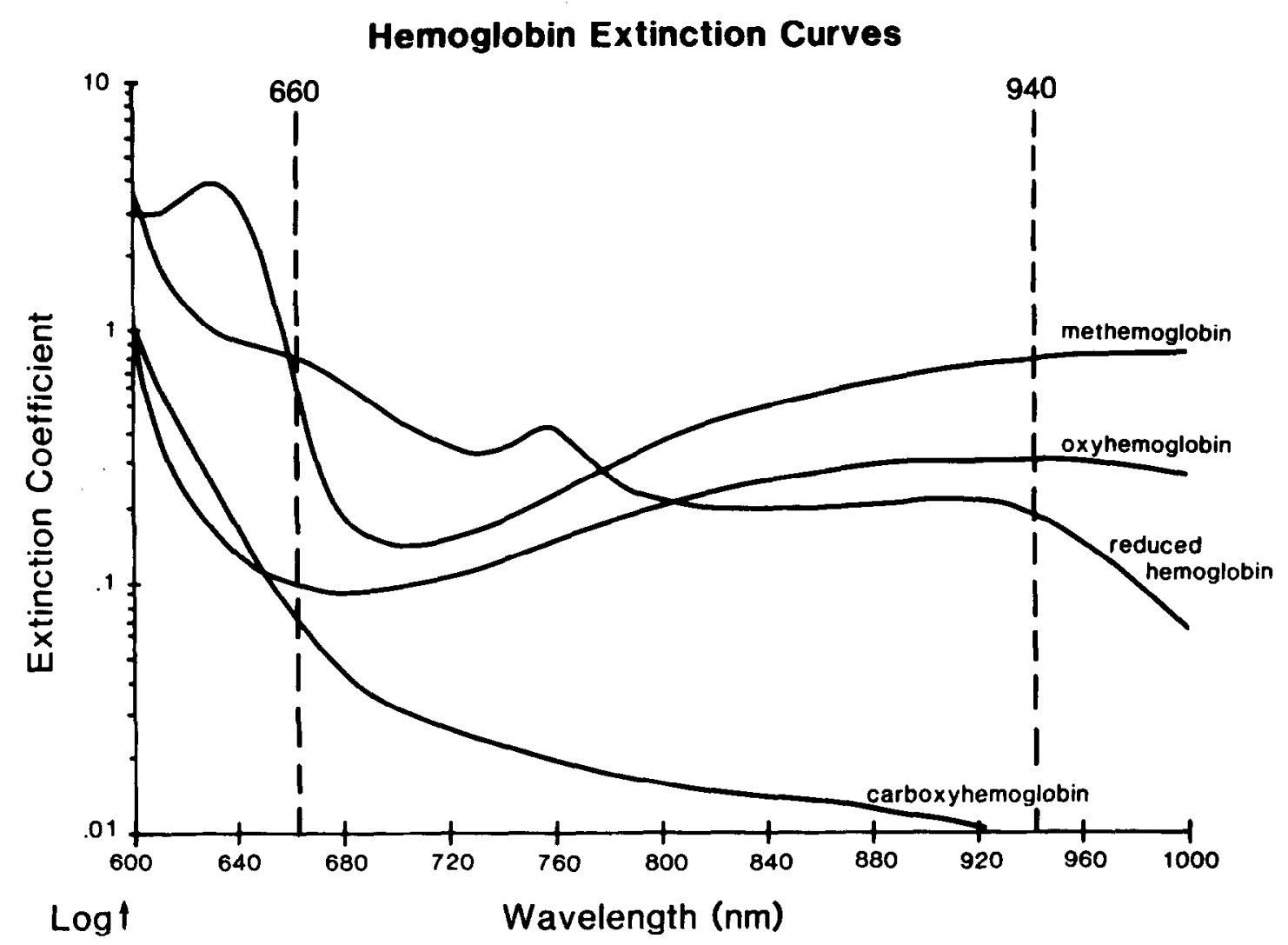

Fig. 4- Transmitted light absorbance spectra of four haemoglobin species: oxyhaemoglobin, reduced haemoglobin, carboxyhaemoglobin, and methaemoglobin. (From: Barker SJ, Tremper KK. Pulse oximetry: applications and limitations. In: Advances in Oxygen Monitoring, International Anesthesiology Clinics. Boston: Little, Brown \& Company, 1987: 155-175, with permission.)

the oxyhaemoglobin dissociation curve of $\mathrm{HbF}$ is shifted to the left from that of $\mathrm{HbA}$, the arterial oxygen tension at a given $\mathrm{SaO}_{2}$ will be lower for $\mathrm{HbF}$. Thus the presence of fetal haemoglobin will affect treatment only if one plans to maintain a specific $\mathrm{PaO}_{2}$ as opposed to a specific $\mathrm{SaO}_{2}$.

Since the photodiode detectors in the pulse oximeter sensor will produce a current from light of any wavelength, ambient light can contaminate the light emitting diode (LED) signal. The pulse oximeter designers have reduced this light interference by alternately turning on the red LED, the infrared LED, and then turning both LEDs off. The photodiode sensor first detects a signal from the red LED plus room light, followed by the infrared LED plus room light, and finally the ambient room light signal alone when both LEDS are turned off. This sequence is repeated 480 times a second in an effort to subtract the room light signal even in a quickly changing light background. Unfortunately, despite this clever design room light interference can cause erroneous $\mathrm{SpO}_{2}$ values or prevent the pulse oximeter from obtaining any $\mathrm{SpO}_{2}$ value. This problcm can be alleviated by covering the sensor with something opaque.

The most difficult problem in the engineering design of the pulse oximeter is the management of low signal to noise ratios. This problem arises from two sources. First, when there is a small absorbance signal due to a weak pulse the device will automatically increase the signal amplification so that it can analyse the pulse-added absorbance signal. Unfortunately, as the signal is amplified the background noise is also amplified. In situations of a very weak or nonexistent pulse signal, the noise can be amplified until it is interpreted by the pulse oximeter as a pulse-added absorbance. New pulse oximeters have signal strength limits, below which they will display a 'low signal-strength' error message. Some devices also display a pulse waveform which can be visually observed to confirm signal quality. Because of its automatic gain control the pulse oximeter can deal with both large and small pulse signals and is relatively insensitive to changes in peripheral pulse amplitude and flow. Lawson et al objectively assessed the blood flow limits of pulse oximeter $\mathrm{SpO}_{2}$ estimation by performing the following experiment. ${ }^{35}$ The peripheral blood flow at the finger was measured by a laser-doppler flow probe which follows changes in red cell flow rate. A pulse oximeter was placed on the finger adjacent to the flow probe while a blood pressure cuff was progressively inflated on the upper arm. The pulse oximeter stopped estimating saturation and gave a 'low perfusion' warning when the peripheral blood flow had decreased to $8.6 \%$ of the control value. When the blood pressure cuff was 
slowly deflated from full occlusion the pulse oximeter again displayed a pulse and saturation estimate when the blood flow as only $4 \%$ of baseline. This study demonstrates the ability of the pulse oximeter to estimate saturation over an extreme range of peripheral blood flow. Clinical and experimental studies have also found that the pulse oximeter can estimate saturation over a wide range of cardiac output as long as an adequate pulse is detected.

Another serious signal to noise problem results from patient motion artifact. For recovery room and the ICU applications this may be the most troublesome pulse oximeter design problem. Most pulse oximeters employ a variety of signal averaging modes to minimize motion effects. That is, the device averages multiple pulses to obtain its saturation estimate, thereby diminishing the effect of single spurious motion signals. Increasing the signal averaging time, however, has a deleterious affect on the time response of the pulse oximeter to acute changes in saturation. The manufacturers can also incorporate more sophisticated algorithms to discriminate motion signals from arterial pulse signals by using the rate of change of $\mathrm{SpO}_{2}$. For example, if the calculated $\mathrm{SpO}_{2}$ changes from $95 \%$ to $50 \%$ in $0.1 \mathrm{~s}$, this new and presumably spurious saturation estimate will either be dropped from the averaging or given a lower weighting factor. As with amplified background noise, motion artifact is probably most reliably detected by direct observation of the plethysmograph waveform.

A final limitation on pulse oximeter accuracy involves the variability of the wavelengths of light emitted by the LEDs. Although these diodes nominally radiate at either $660 \mathrm{~nm}$ or $940 \mathrm{~nm}$, in reality each diode emits a slightly different wavelength. The peak or centre wavelength of light from the LED can vary $\pm 10 \mathrm{~nm}$ from the specified value. A shift in the light emitting diode wavelength will change the absorbance coefficient. Since the oximeter software assumes an absorbance coefficient at a specified wavelength, an unknown change in that wavelength will cause an error in the saturation estimate. Therefore in reality there is a probe-toprobe variability in accuracy. Some manufacturers attempt to minimize this error by narrowing the wavelength tolerance on the LEDs used in their sensors. Others compensate for this error by designing the sensor to identify its specific wavelengths to the pulse oximeter monitor, which then electronically corrects for shifted centre wavelengths.

Accuracy and response. Most clinical pulse oximeters have a specified accuracy of $\pm 2 \%$ from $100 \%$ down to $70 \%$ saturation, $\pm 3 \%$ from $70 \%$ saturation to $50 \%$, and no unspecified accuracy below $50 \%$ saturation. ${ }^{34,36}$ This implies that the $\mathrm{SpO}_{2}$ is within $2 \%$ above or below the actual $\mathrm{SaO}_{2} 67 \%$ of the time $( \pm 1$ standard deviation around the mean). This corresponds to a $99 \%$ confidence interval of $\pm 6 \%$ (i.e. 3 standard deviations) from $100 \%$ to $70 \%$ saturation, and $\pm 9 \%$ from $70 \%$ to $50 \%$ saturation. Although these manufacturer's specifications are based on volunteer data collected under optimal conditions, the accuracies found in clinical studies are comparable. It is often difficult to compare clinical studies to manufacturer's specifications, because the studies usually report correlation coefficients and linear regression analysis rather than the more appropriate bias and precision. From the available studies on adult, paediatric and neonatal patients it appears that under steady-state conditions the pulse oximeter $\mathrm{SpO}_{2}$ value will be within \pm 2 to 3 saturation points ( \pm 1 standard deviation). Experimental studies in adult volunteers evaluated the response time of pulse oximeters to sudden desaturation and resaturation. ${ }^{37}$ Severinghaus and Naifeh also found that the ear probe responds more quickly than the finger probe during desaturation and resaturation. ${ }^{37}$ Their $\mathrm{T}^{1 / 2}$ values for ear probes ranged from 9.6-19.8s while the $T^{1 / 2}$ for finger probes ranged from $24-35.1 \mathrm{~s}$.

Considering all the sources of possible error, pulse oximeters are impressively accurate clinical devices. Since the manufacturers' specified accuracy of \pm 2 to $3 \%$ is only for a $68 \%$ confidence interval, it is surprising that clinical studies have found accuracies similar to these.

\section{Noninvasive haemoglobin saturation monitoring: tissue oximetry}

Near infrared light penetrates most tissues including bone but is absorbed and reflected by haemoglobin and cytochrome aa3. The absorbance or reflectance of infrared light by both of these substances is oxygen saturation dependent. ${ }^{38}$ If tissues are illuminated with infrared light, the transmitted or reflected intensity will change in relation to the overall saturation of both haemoglobin and cytochrome aa 3 and also the absolute amount of the absorbers in the volume of tissue involved. Therefore, this 'non-pulse' oximetry may provide an estimate of the mean tissue saturation. The pioneering work in this field done by Frans Jobsis was primarily focused on the measurement of cytochrome aa3 saturation. ${ }^{39}$ More recently, effort has been focused on the global values obtained when cerebral tissues are illuminated. ${ }^{38}$ In most tissues the majority of blood resides in the venous system, therefore, the information provided from tissue illumination predominantly reflects the venous tissue blood saturation.

As discussed earlier in the section on Beer's law the pathlength of light is critical in determining the concentration measurement. With reflected tissue saturation measurements, the pathlength is very ill-defined. Once the light enters the tissue there is substantial light scattering which dramatically affects the pathlength. In addition, since reflected light signal is being measured the pathlength is affected by the depth of penetration. Current work is underway 
to try to account for these effects and, thereby, develop a technique which will provide quantitative data. Current techniques can rapidly follow changes in brain 'tissue saturation' or blood volume but reproducible quantitative data are more elusive. Nevertheless, this technique does provide a measurement of the most basic variable of interest with respect to oxygen monitoring the tissue.

\section{Summary}

Over the past 2 decades, the principles involved in laboratory oxygen measurements have been applied to an array of invasive and noninvasive continuous monitoring techniques. Each of the techniques provides different information regarding the oxygen transport system due to the fact that it is measuring a different variable by a different process at a different location along the oxygen transport system. The availability of continuous oxygen monitoring enables us to improve our patient care only if the information provided is interpreted properly. With a basic understanding of how these devices work, the data they present may be more accurately interpreted.

\section{References}

1. Tremper KK. Advances in Oxygen Monitoring. International Anesthesia Clinics. Boston: Little, Brown and Company, Winter, 1987.

2. Clark LC Jr. Monitor and control of tissue oxygen tensions. Trans Am Soc Artificial Internal Organs 1956; 2: 41.

3. Severinghaus JW, Bradley AF. Electrodes for blood $\mathrm{PO}_{2}$ and $\mathrm{PCO}_{2}$ determination. J Appl Physiol 1958; 13: 515.

4. Tremper KK, Barker SJ. Fundamental principles of monitoring instrumentation. In: Miller R, ed. Anesthesia, 3rd ed. New York: Churchill-Livingstone Inc, 1990: 957-999.

5. Gehrich JL, Lubbers DW, Opitz $\mathrm{N}$ et al. Optical fluorescence and its application to an intravascular blood gas monitoring system. IEEE Transactions on Biomedical Engineering 1986; BME-33: 117-132.

6. Optiz N, Lubbers DW. Theory and development of fluorescent based photochemical oxygen sensors: oxygen optodes. Int Anesthesiol Clin 1987; 25: 177-197.

7. Peterson JL, Vurek GG. Fiberopotic sensors for biomedical applications. Science 1984; 224: 123-127.

8. Lippitsch ME, Pusterhofer J, Leiner MJ, Wolfbeis OS. Fiberoptic oxygen sensor with fluorescence decay time as information carrier. Analytica Chimica Attica 1988; 205: 1-6.

9. Wahr J, Tremper KK. Continuous intravascular blood gas monitoring: state of the art. J Cardiothorac Vasc Anesth (in press).

10. Kollmeyer KR, Tsang RC. Complications of umbilical oxygen electrodes. J Pediatr 1974; 84: 894-897.

11. Bralanow N, Pulk K, Bland R, Kram HB, Lee TS, Shoemaker WC. Continuous polarographic monitoring of intra-arterial oxygen in the perioperative period. Crit Care Med 1985; 13: 859-860.

12. Malalis L, Bhat R, Vidyasagnar D. Comparison of intravascular $\mathrm{PO}_{2}$ with transcutaneous and $\mathrm{PaO}_{2}$ values. Crit Care Med 1983; 11: 110-113.

13. Bland JM, Altman DG. Statistical methods for assessing agreement between two methods of clinical measurement Lancet 1986; $1: 307-310$.

14. Shapiro BA, Harrison RA, Cane RD et al. Quality assurance in blood gas analysis. In: Shapiro BA, ed. Clinical Application of Blood Gases, 4th ed. Chicago: Year Book Medical Publishers, Inc. 1989; 274-282.
15. Mahutte CK, Sassoon SH, Muro JR et al. Progress in the development of a fluorescent intravascular blood gas system in man. J Clin Monit 1990; 6: 147-157.

16. Barker SJ, Hyatt J. Continuous measurement of intraarterial $\mathrm{pHa}, \mathrm{PaCO}_{2}$, and $\mathrm{PaO}_{2}$ in the operating room. Anesth Analg 1991; 73: 43-48.

17. Shapiro BA, Mahutte CK, Cane RD, Gilmour IJ. Clinical performance of a blood gas monitor: A prospective, multicenter trial. Crit Care Med 1993; 21: 487-494.

18. Zimmerman JL, Dellinger RP. Initial evaluation of a new intra-arterial blood gas system in humans. Crit Care Med 1993; 21: 495-500.

19. Zimmerman JL, Dellinger RP. Evaluation of a continuous intra-arterial blood gas system in critically ill patients. Chest 1992; 102(2): 105S.

20. Greenblott GB, Tremper KK, Barker SJ, Gerschultz S, Gehrich JL. Continuous blood gas monitoring with an intraarterial optode during one-lung anesthesia. $J$ Cardiothorac Vasc Anesth 1991; 5(4): 365-367.

21. Greenblott GB, Barker SJ, Tremper KK, Gerschultz S, Gehrich JL. Detection of venous air embolism by continuous intraarterial oxygen monitoring. J Clin Monit 1990; 6(1): 53-56.

22. Tremper KK. Transcutaneous $\mathrm{PO}_{2}$ measurement. Canadian Anesthetist Society Journal 1984; 31: 664-677.

23. Glenski JA, Cucchiara RF. Transcutaneous $\mathrm{O}_{2}$ and $\mathrm{CO}_{2}$ monitoring of neurosurgical patients: Detection of air embolism. Anesthesiol 1986; 64: 546-550.

24. Abraham E, Oye RK, Smith M. Detection of blood volume deficits through conjunctival oxygen tension monitoring. Crit Care Med 1985; 12: 931-934.

25. Martin WE, Cheung PW, Johnson CC, Wong KC. Continuous monitoring of mixed venous oxygen saturation in man. Anesth Analg 1973; 52: 784-793.

26. Wilkinson AR, Phibbs RH, Gregory GA. Continuous measurement of oxygen saturation in sick newborn infants. J Pediatr 1978; 93: 1016-1019.

27. Baele PL, McMichan JC, Marsh HM, Sill JC, Southorn PA. Continuous monitoring of mixed venous oxygen saturation in critically ill patients. Anesth Analg 1982; 61(6): 513-517.

28. Barker SJ, Tremper KK, Hyatt J, Zaccari J. Effects of methemoglobinemia on pulse oximetry and mixed venous oximetry. Anesthesiol 1987; 67: A170.

29. Haney M, Tremper KK. Effect of carboxyhemoglobin on the accuracy of mixed venous oximetry monitors in dogs. Crit Care Med 1993; (in press).

30. Lee SE, Tremper KK, Barker SJ. Effects of anemia on pulse oximetry and continuous mixed venous oxygen saturation monitoring in dogs. Anesthesiol 1991; 118-122.

31. O'Connor JP, Townsen GE. Routine perioperative monitoring of mixed venous saturation pro: perioperative continuous monitoring and mixed venous oxygen saturation should be routine during high risk surgery. J Cardiothorac Anesth 1990; 4: 647-650.

32. Boylan JF, Teasdle SJ. Routine perioperative monitoring and mixed venous oxygen saturation con: perioperative continuous monitoring of mixed venous oxygen saturation should be routine during high risk cardiac surgery patients. J Cardiothorac Anesth 1990; 4: 651-654.

33. American Society of Anesthesiologists. Standards for basic intraoperative monitoring. Anesthesia Patient Safety Foundation 1987: March 3.

34. Tremper KK, Barker SJ. Pulse oximetry. Anesthesiol 1989; 70: $98-108$.

35. Lawson D, Norley I, Korbon G, Loeb R, Ellis J. Blood flow limits and pulse oximeter signal detection. Anesthesiol 1987; 67: $599-603$.

36. Severinghaus JW, Kelleher JF. Recent developments in pulse oximetry. Anesthesiol 1992; 76: 118-138.

37. Severinghaus JW, Naifeh KH. Accuracy of response of six pulse oximeters to profound hypoxia. Anesthesiol 1987;67: 551-558.

38. Kurth CD, Steven JM, Benarom D, Chance B. Near-infrared monitoring of the cerebral circulation. J Clin Monit 1993; 9: $163-170$.

39. Inhsis FF. Noninvasive, infrared monitoring of cerebral and myocardial oxygen sufficiency and circulatory patterns. Science 1977; 198: 1264-1267. 